\title{
Backscattering suppression in supersonic 1D polariton condensates
}

\author{
D. Tanese ${ }^{1}$ D. D. Solnyshkov ${ }^{2}$ A. Amo,${ }^{1}$ L. Ferrier,${ }^{1}$ E. Bernet-Rollande,${ }^{1}$ \\ E. Wertz, ${ }^{1}$ I. Sagnes, ${ }^{1}$ A. Lemaitre, ${ }^{1}$ P. Senellart,${ }^{1}$ G. Malpuech, ${ }^{2}$ and J. Bloch ${ }^{1}$ \\ ${ }^{1}$ CNRS, Laboratoire de Photonique et Nanostructures, Route de Nozay, 91460 Marcoussis, France \\ ${ }^{2}$ LASMEA, Clermont Université, University Blaise Pascal, \\ CNRS, 24 avenue des Landais, 63177 Aubière cedex, France
}

(Dated: April 13, 2017)

\begin{abstract}
We investigate the effects of disorder on the propagation of one-dimensional polariton condensates in semiconductor microcavities. We observe a strong suppression of the backscattering produced by the imperfections of the structure when increasing the condensate density. This suppression occurs in the supersonic regime and is simultaneous to the onset of parametric instabilities which enable the "hopping" of the condensate through the disorder. Our results evidence a new mechanism for the frictionless flow of polaritons at high speeds.

PACS numbers: 71.36.+c, 67.10.Jn, 78.67.De, 42.65.Wi
\end{abstract}

The interplay between kinetic energy, localization energy, and particle interactions lies at the heart of the transport properties in condensed matter physics. This problem has been thoroughly treated theoretically since the seminal work of Anderson [1, 2], which described the localization of electrons in disordered media caused by the interference between the electronic wavefunctions.

Electron localization phenomena have been observed in doped semiconductors [3], but this systems suffers from a large number of non-controllable parameters, like the strong Coulomb interactions or the disorder potential. As an alternative, gases of neutral bosons appear as model systems for the understanding of localization. While some works have addressed these questions in the eighties [4], this activity took an enormous theoretical and experimental expansion since the observation of BoseEinstein condensates of ultracold atoms [5], and has allowed the study of localization at low dimensions in controlled disorder landscapes. One dimensional systems are of particular interest as the reduction of the available scattering channels enhances the interference effects. Formally, this gives rise to localization for any disorder strength [6]. Recent experiments have indeed shown the localization of bosonic matter waves in the presence of random disorder using ultracold atoms $[7,[\underline{8}]$.

While disorder tends to localize the wavepackets, interparticle repulsive interactions have a delocalization effect. A localized to extended phase transition has been predicted to be triggered by the repulsion between particles in 1D [9, 10], and recent experiments have demonstrated this effect in atomic condensates at rest [11]. Particularly interesting is the case of a 1D boson flow in the presence of a weak disordered potential. If the considered sample is small enough and/or the disorder is weak, a quasi-extended state is recovered with a high transmission [12], still with an appreciable backscattering amplitude. Repulsive interactions in such a flowing boson gas could lead to the formation of a superfluid state, resulting in the perfect transmission through the $1 \mathrm{D}$ channel [9].
However, the superfluid behaviour is lost if the flow speed becomes larger than a critical velocity [13] which is close to the speed of sound of the fluid given by $c=\sqrt{\alpha n / m}$, where $m$ is the mass of the particles, $n$ is their density, and $\alpha$ the interparticle interaction energy. Nevertheless, as we will see in this work, even at high flow speeds in the non-superfluid regime, interactions between particles can lead to a strong reduction of the backscattering and, consequently, to an enhancement of the transmission.

In this letter we study the effects of interparticle interactions on the propagation of a polariton boson condensate in a $1 \mathrm{D}$ semiconductor wire microcavity. We observe, in the supersonic regime, a strong suppression of the signal backscattered by the imperfections of the structure while increasing the condensate density. This suppression, which was previously theoretically predicted in [9], is caused by the onset of parametric instabilities arising from particle interactions, giving the condensate access to propagating states which bypass the potential barriers caused by disorder. This confirms experimentally the existence of a new mechanism for the suppression of the backscattering, very different from the recently reported superfluidity of polaritons [14, 15].

Cavity polaritons are bosonic particles arising from the strong coupling between quantum well excitons and photons confined in a microcavity. Their Bose-Einstein condensation has been reported by several groups 16 19]. Non-linear phenomena such as optical parametric oscillation (OPO) 20, 21], multistable behaviour 22], and superfluidity 15, 23 have been demonstrated. Recent technological breakthroughs have allowed the production of very high quality microcavities with polariton lifetimes as long as 30 ps -5 times larger than in the pre-existing samples. In a $1 \mathrm{D}$ geometry, this achievement has allowed the observation of propagating polariton condensates spatially separated from the pumping area and showing phase coherence extending over one hundred healing lengths [24]. Here we use this geometry to study the effect of interactions on the backscattering 


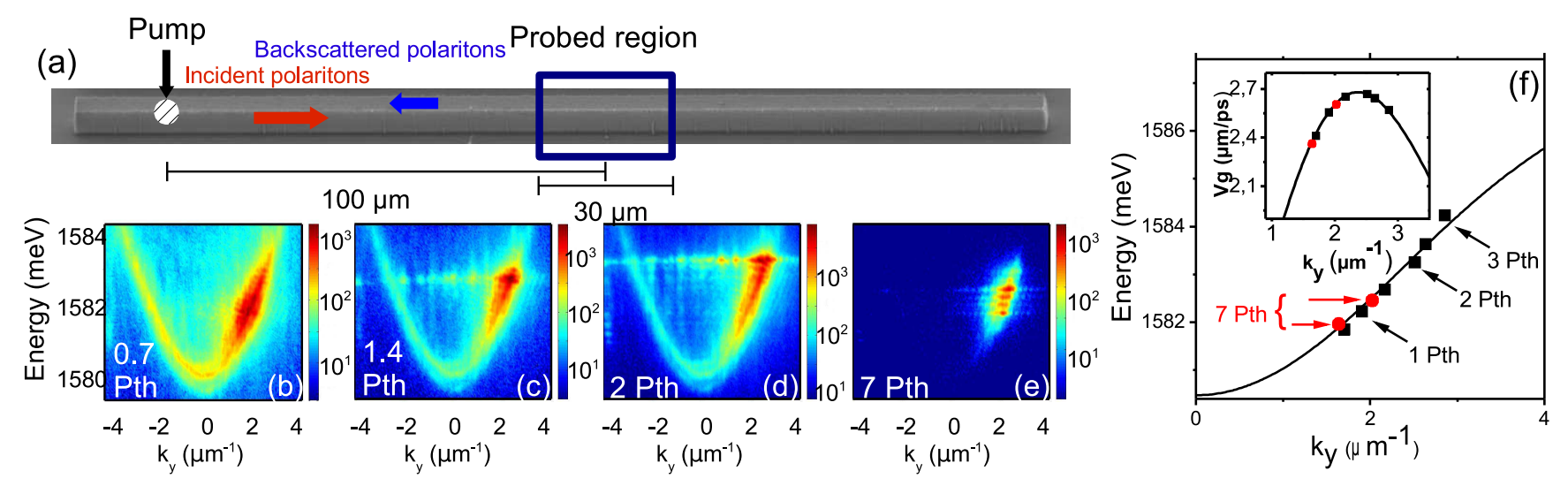

FIG. 1. (Color online) (a) Scanning electron microscope image of the wire and scheme of the experiment. (b)-(e) Normalised far field emission of the polariton gas in the probed region. (f) Energy and momentum of the polariton condensates at different excitation powers, below (black squared points) and above (red round points) the onset for stimulated parametric processes. The solid line shows the polariton dispersion and the inset the corresponding values of the group velocity.

caused by imperfections in the structure.

Our samples are GaAs/AlGaAs based microcavities etched into $1 \mathrm{D}$ wires with a width of $3.5 \mu \mathrm{m}$ and a length of $0.2 \mathrm{~mm}$ [24]. The Rabi splitting amounts to $15 \mathrm{meV}$ and the polariton lifetime to $30 \mathrm{ps}$. Polariton condensates are formed under non-resonant photoexcitation (laser wavelength around $730 \mathrm{~nm}$ ) with a singlemode Ti:Sapph laser. The laser spot has a $2 \mu \mathrm{m}$ diameter and is positioned close to one of the edges of the wire [see Fig. 1(a)]. At low excitation density, carriers are photoinjected at the position of the excitation spot, relaxing down to form polaritons which populate the lower polariton branch. This incoherent gas of polaritons with different momenta can propagate long distances in the wire thanks to the long cavity lifetime. Figure 1(b) shows the momentum space in logarithmic scale of the polariton signal $100 \mu \mathrm{m}$ away from the injection area. For positive values of the momentum, the forward propagating polaritons can be observed. Due to the large value of the exciton mass, the population of the excitonic reservoir is negligible out of the excitation area.

Above a threshold excitation density $P_{t h}$ [Fig. 2(a)], polariton condensation takes place at $k=0$ in the region optically pumped by the laser. In that region, the presence of a dense excitonic reservoir induces a strong blueshift of the polariton energy even at few times $P_{t h}$, as discussed in Ref. 24]. The energy difference between the $k=0$ states in and out of the pumped area results in an acceleration of the polariton condensate from the pump spot. As a result, in the region of observation, the propagating condensate preserves the same emission energy as in the pumped region but it gains a finite momentum [2.1 $\mu \mathrm{m}^{-1}$ in Fig. 11(c)]. By further increasing the polariton density, the reservoir in the pumped area induces a larger blueshift and the propagating condensate emits at higher energy with a larger $k$, as shown in (d) and in the black dots of Fig. 1(f).

This progression continues while increasing the excitation density until the propagating condensate gets close to the inflexion point of the lower polariton branch. Then, parametric instabilities induced by polariton interactions set up the spontaneous oscillation of the polariton emission between several coexisting states with different energies [see Fig. [1(e)]. Note that contrary to the case in non-linear crystals or in previous OPO experiments in polaritons [20, 21], none of the states in the OPO is resonantly fed by a laser source. Here, the OPO is spontaneously triggered once a sufficiently high population is accumulated in the propagating condensate. This is a remarkable example of a self-sustained optical parametric oscillator. As we will see below, these parametric instabilities play an important role in the quenching of the polariton backscattering.

Let us now concentrate on the scattering of the propagating polariton gas with the weak disorder present in the wire microcavity. The origin of the disorder is structural and gives rise to a change of the polariton energy smaller than $0.1-0.4 \mathrm{meV}$. It is mainly caused by the weak strain accumulated during the growth of the mirrors and also to the non-intentional rugosity that is formed on the walls of the wires during the lithographic and etching processes. Figure1(b) shows a significant signal backscattered from the incoherent polariton gas at negative momenta. The origin of this signal in the far field can only arise from the scattering of propagating polaritons with disorder as those states cannot be fed by the excitonic reservoir, located $100 \mu \mathrm{m}$ away from the observation area. When the polariton density is increased, the relative backscattered signal is strongly reduced, as shown in Fig. 1(b)-(e) and summarized in Fig. 2(b). The quenching of the backscattering reaches a factor of 20 at the highest investigated excitation density, with respect to the low density regime. 


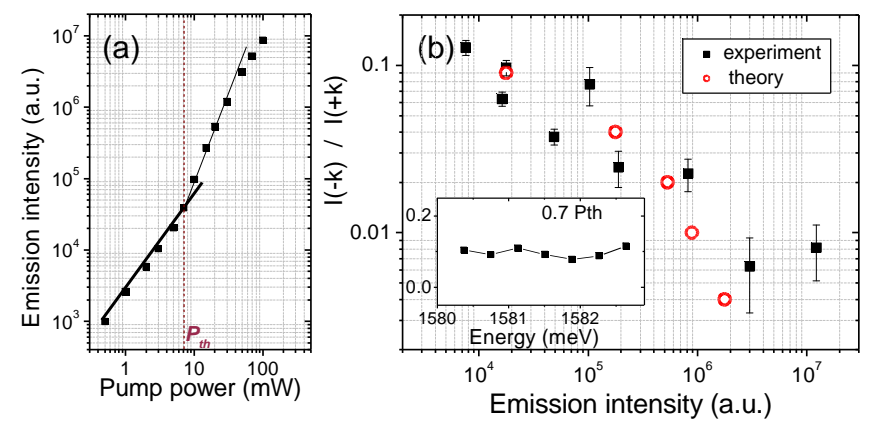

FIG. 2. (Color online) (a) Emitted intensity in the probed region as a function of excitation power. (b) Black points (red circles): ratio of the backscattered $[I(-k)]$ to the incident polariton signal $[I(+k)]$ in the probed region measured at the energy of the peak signal, experimentally (theoretically) obtained in the conditions of Fig. 1] Inset: backscattered ratio measured at low excitation power (below the condensation threshold) as a function of the energy of the propagating polaritons.

This remarkable reduction is not caused by the increase of the kinetic energy with excitation power, since the intrinsic backscattered amplitude, measured at low density, does not depend on the polariton energy [see inset of Fig. 2(b)]. The origin of the strong reduction of the scattering can neither be in a transition to a superfluid regime at high density in the context of the Landau criterion, as recently reported in two dimensions under resonant excitation [14, 15]. Our condensates travel at large speeds, with momenta on the order of $2-3 \mu \mathrm{m}^{-1}$ and energy $\sim 3 \mathrm{meV}$ above the $k=0$ state. In order to reach the superfluid regime, interaction induced blueshifts at least twice the value of the kinetic energy would be required. We are far from that situation, meaning that our fluids are supersonic.

We propose a novel mechanism for the quenching of the backscattering based on the onset of spontaneous parametric processes as the polariton density is increased. In order to understand this mechanism, let us go back to the Anderson model of localization by disorder [1]. One of its simplest descriptions is to consider a regular 1D lattice in the tight-binding model with a hopping constant $J$, the energy of each site being random [see Fig. 3(a)]. If the energy difference between two sites $\left(E_{1}, E_{2}\right)$ is smaller than $J\left(\left|E_{1}-E_{2}\right|<J\right)$, the particle can jump from one site to the other. If the probability of finding an available neighbor for the jump is $P(<1)$, then the probability to make $N$ jumps will be $P^{N}$, which gives an exponentially decaying distribution around the initial site.

Let us consider now a system with local repulsive interaction between particles. This interaction can shift the on-site energy, increasing the probability to match the energies of two different sites. Additionally, the onset of OPO on-site scattering can populate virtually any energy state via the following energy conserving process:
$E_{1}+E_{1} \rightarrow\left(E_{1}+\Delta\right)+\left(E_{1}-\Delta\right)$, where $2 \Delta$ is the energy difference between signal and idler states. If the condition $\left|E_{1} \pm \Delta-E_{2}\right|<J$ is verified, a particle can jump to the neighboring site [Fig. [3(a)].

In our experiment the kinetic energy of particles is much larger than the localization energy. Therefore, the effect of local blueshift is expected to be weak compared to that of the onset of parametric instabilities, which increases the probability of finding a neighbor with a matching energy and tends to suppress localization. This phenomenon has been theoretically discussed in Ref. 10] in the context of ultracold atoms, while a similar effect has been addressed within a different approach in Ref. [9]. In the latter case, calculations showed that because of the interactions the decay of the transmission of a 1D atomic Bose-Einstein condensates through a disordered waveguide is algebraic instead of exponential, a signature of the suppression of Anderson localization. This regime corresponds to "non-stationary" solutions of the GrossPitaevskii equation, evidencing the presence of manyenergy states in the system, in line with our interpretation of the onset of parametric processes in experiments.

In order to support our model for the quenching of the backscattering, we have performed numerical simulations of the propagation of polariton condensates with a certain wave-vector through a series of point-like defects. To describe accurately the polariton condensate, we use a set of Schrödinger equations describing the time evolution of the photonic $\psi_{p h}(x, t)$ and excitonic $\psi_{e x}(x, t)$ mean fields coupled via the light matter interaction (Rabi splitting, $\Omega_{R}=15 \mathrm{meV}$ ). Our model takes into account the two allowed spin projections of the photon and exciton fields $\sigma= \pm 1[25]$ :

$$
\begin{aligned}
i \hbar \frac{\partial \psi_{p h}^{\sigma}}{\partial t} & =-\frac{\hbar^{2}}{2 m_{p h}} \Delta \psi_{p h}^{\sigma}+\frac{\Omega_{R}}{2} \psi_{e x}^{\sigma} \\
& -\frac{i \hbar}{2 \tau_{p h}} \psi_{p h}^{\sigma}+P^{\sigma}+H_{e f f} \psi_{p h}^{-\sigma}+U \psi_{p h}^{\sigma} \\
i \hbar \frac{\partial \psi_{e x}^{\sigma}}{\partial t} & =-\frac{\hbar^{2}}{2 m_{e x}} \Delta \psi_{e x}^{\sigma}+\frac{\Omega_{R}}{2} \psi_{p h}^{\sigma} \\
& -\frac{i \hbar}{2 \tau_{e x}} \psi_{e x}^{\sigma}+\alpha\left|\psi_{e x}^{\sigma}\right|^{2} \psi_{e x}^{\sigma}
\end{aligned}
$$

Here $m_{p h}=3.6 \times 10^{-5} m_{0}, m_{e x}=0.4 m_{0}$ and $m_{0}$ are the cavity photon, the quantum well exciton and the free electron masses, respectively. The lifetime of the particles are $\tau_{p h}=15 \mathrm{ps}$ and $\tau_{e x}=400 \mathrm{ps}$. $H_{\text {eff }}$ accounts for the TE-TM polarization splitting of the waveguided modes. $\alpha=6 E_{b} a_{B}^{2} / S$ is the polariton-polariton interaction constant $\left(E_{b}=6 \mathrm{meV}\right.$ is the exciton binding energy, $a_{B}=10 \mathrm{~nm}$ is the exciton Bohr radius), $U(x)$ is the disorder potential along the wire which is a series of randomly spaced delta peaks [shown in Fig. [3 (b)], $P(x, t)$ is the coherent $c w$ pumping, localized on a spot of $2 \mu \mathrm{m}$ 


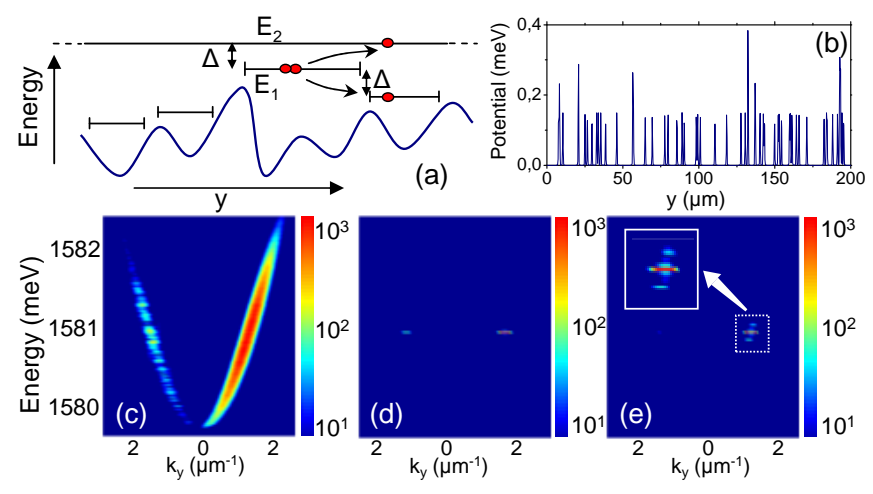

FIG. 3. (Color online) (a) Schematic representation of the disorder potential showing the spatial extension of localised states (horizontal bars) and the parametrically induced hopping process. (b) Disorder potential used in the model. (c)(e) Simulation of the polariton far field emission in the disordered potential with increasing polariton density. The upper left squared region in (e) shows a magnification of the lower squared area.

placed on the left of the sample, as sketched on Fig. 1(a), injecting polaritons with well defined momenta.

Figures 3(c)-(e) show the far field emission $100 \mu \mathrm{m}$ away from the excitation spot obtained from the simulations at different excitation densities. To simulate the situation below threshold [Fig. 3(c)], we inject a short pulse, filling all positive momenta states of the lower polariton branch with a distribution similar to the measured one [Fig. 1(b)]. A strong backscattered signal can be observed, analogous to the experimental one shown in Fig. 1(b). Note that the backscattering is entirely due to the presence of the disorder potential. Just above threshold the condensate presents a single energy [Fig. 1(c)], which we simulate by quasiresonant $c w$ pumping with a well defined $k$ [Fig. 3(d)]. A significant backscattered signal is still present. At higher densities [Fig. 3(e)], new propagation frequencies associated with the onset of parametric processes become clearly visible, whereas the pumping is still monochromatic. The well-defined separation between the frequencies, which can be observed both in the experimental [Fig. 1(d)] and theoretical images [Fig. 3(e)], is due to the polarization splitting $2 H_{\text {eff }} \approx 0.5 \mathrm{meV}$. It determines the preferential resonances, for the onset of the OPO processes. This model provides an interpretation to the observation of apparently spectrally equidistant condensates 24] at high pumping rates. The energy spacing between the different frequencies is in our approach not related to a balance between scattering rates and losses [26], but to the value of the TE-TM splitting.

Similar to the results of Ref. [9], the appearance of the new frequencies results in the reduction of the backscattered signal. Figure 2(b) summarizes in open dots the calculated backscattered intensity averaged over 50 dis- order realisations, as a function of the excitation density. They show a reduction by more than one order of magnitude when the density is increased by a factor of 100 , in good agreement with the experimental results. This value is significantly greater than the one found in experiments of superfluidity in planar microcavities (a quenching by a factor of 4) 15]. Though backscattering effects are less important in 2D than in $1 \mathrm{D}$ systems due to the very different density of available states in each case, the mechanism we propose might as well play a role on the flow of polaritons in triggered optical parametric configurations in planar microcavities [23, 27].

In conclusion, we have observed a strong suppression of the scattering in propagating $1 \mathrm{D}$ polariton condensates caused by the onset of parametric instabilities at high excitation densities, which enable the "hopping" of the condensate through the disorder. This new mechanism results in a quasi-frictionless flow of supersonic polariton condensates, opening the way to the fabrication of integrated polariton circuits with high transmissivity.

We are greatful to A. V. Kavokin, P. Leboeuf and N. Pavloff for fruitful discussions. This work was partly supported by the C'Nano Ile de France contract "Sophiie2", the RTRA contract "Picorre", and the FP7 ITNs "Clermont4" (235114) and "SpinOptronics"(237252).

[1] P. W. Anderson, Phys. Rev. 109, 1492 (1958).

[2] A. Lagendijk, B. van Tiggelen, and D. S. Wiersma, Physics Today 62, 24 (2009).

[3] T. F. Rosenbaum, R. F. Milligan, M. A. Paalanen, G. A. Thomas, R. N. Bhatt, and W. Lin, Phys. Rev. B 27, 7509 (1983).

[4] M. P. A. Fisher, P. B. Weichman, G. Grinstein, and D. S. Fisher, Phys. Rev. B 40, 546 (1989).

[5] M. H. Anderson, J. R. Ensher, M. R. Matthews, C. E. Wieman, and E. A. Cornell, Science 269, 198 (1995); K. B. Davis, M. O. Mewes, M. R. Andrews, N. J. van Druten, D. S. Durfee, D. M. Kurn, and W. Ketterle, Phys. Rev. Lett. 75, 3969 (1995).

[6] N. F. Mott and W. D. Twose, Adv. Phys. 10, 107 (1961).

[7] J. Billy, V. Josse, Z. Zuo, A. Bernard, B. Hambrecht, P. Lugan, D. Clement, L. Sanchez-Palencia, P. Bouyer, and A. Aspect, Nature 453, 891 (2008).

[8] G. Roati, C. D'Errico, L. Fallani, M. Fattori, C. Fort, M. Zaccanti, G. Modugno, M. Modugno, and M. Inguscio, Nature 453, 895 (2008).

[9] T. Paul, P. Leboeuf, N. Pavloff, K. Richter, and P. Schlagheck, Phys. Rev. A 72, 063621 (2005); T. Paul, M. Albert, P. Schlagheck, P. Leboeuf, and N. Pavloff, ibid. 80, 033615 (2009).

[10] I. L. Aleiner, B. L. Altshuler, and G. V. Shlyapnikov, Nature Phys. 6, 900 (2010).

[11] B. Deissler, M. Zaccanti, G. Roati, C. D'Errico, M. Fattori, M. Modugno, G. Modugno, and M. Inguscio, Nature Phys. 6, 354 (2010).

[12] J. B. Pendry, Adv. Phys. 43, 461 (1994). 
[13] S. Ianeselli, C. Menotti, and A. Smerzi, J. Phys. B: At. Mol. Opt. Phys. 39, S135 (2006).

[14] I. Carusotto and C. Ciuti, Phys. Rev. Lett. 93, 166401 (2004).

[15] A. Amo, J. Lefrère, S. Pigeon, C. Adrados, C. Ciuti, I. Carusotto, R. Houdré, E. Giacobino, and A. Bramati, Nature Phys. 5, 805 (2009).

[16] J. Kasprzak, M. Richard, S. Kundermann, A. Baas, P. Jeambrun, J. M. J. Keeling, F. M. Marchetti, M. H. Szymanska, R. Andre, J. L. Staehli, V. Savona, P. B. Littlewood, B. Deveaud, and L. S. Dang, Nature 443, 409 (2006).

[17] R. Balili, V. Hartwell, D. Snoke, L. Pfeiffer, and K. West, Science 316, 1007 (2007).

[18] S. Christopoulos, G. Baldassarri Hoger von Hogersthal, A. J. D. Grundy, P. G. Lagoudakis, A. V. Kavokin, J. J. Baumberg, G. Christmann, R. Butte, E. Feltin, J.-F. Carlin, and N. Grandjean, Phys. Rev. Lett. 98, 126405 (2007).

[19] E. Wertz, L. Ferrier, D. D. Solnyshkov, P. Senellart, D. Bajoni, A. Miard, A. Lemaitre, G. Malpuech, and J. Bloch, Appl. Phys. Lett. 95, 051108 (2009).

[20] R. M. Stevenson, V. N. Astratov, M. S. Skolnick, D. M. Whittaker, M. Emam-Ismail, A. I. Tartakovskii, P. G.
Savvidis, J. J. Baumberg, and J. S. Roberts, Phys. Rev. Lett. 85, 3680 (2000).

[21] C. Diederichs, J. Tignon, G. Dasbach, C. Ciuti, A. Lemaître, J. Bloch, P. Roussignol, and C. Delalande, Nature 440, 904 (2006).

[22] T. K. Paraïso, M. Wouters, Y. Leger, F. MourierGenoud, and B. Deveaud-Plédran, Nature Mat. 10, 80 (2011).

[23] A. Amo, D. Sanvitto, F. P. Laussy, D. Ballarini, E. del Valle, M. D. Martín, A. Lemaitre, J. Bloch, D. N. Krizhanovskii, M. S. Skolnick, C. Tejedor, and L. Viña, Nature 457, 291 (2009).

[24] E. Wertz, L. Ferrier, D. D. Solnyshkov, R. Johne, D. Sanvitto, A. Lemaitre, I. Sagnes, R. Grousson, A. V. Kavokin, P. Senellart, G. Malpuech, and J. Bloch, Nature Phys. 6, 860 (2010).

[25] I. A. Shelykh, Y. G. Rubo, G. Malpuech, D. D. Solnyshkov, and A. Kavokin, Phys. Rev. Lett. 97, 066402 (2006).

[26] M. Wouters, T. C. H. Liew, and V. Savona, Phys. Rev. B 82, 245315 (2010).

[27] D. Sanvitto, F. M. Marchetti, M. H. Szymanska, G. Tosi, M. Baudisch, F. P. Laussy, D. N. Krizhanovskii, M. S. Skolnick, L. Marrucci, A. Lemaitre, J. Bloch, C. Tejedor, and L. Vina, Nature Phys. 6, 527 (2010). 